\title{
Correction to: DNA Cell Biol 2017;36(10):813-821. DOI: 10.1089/dna.2017.3790
}

N THE October 2017 issue of DNA and Cell Biology (vol. 36, no. 10, pp.813-821, DOI: 10.1089/dna.2017.3790), in the article entitled "Trifluoperazine Activates FOXO1-Related Signals to Inhibit Tumor Growth in Hepatocellular Carcinoma" by Jingwen Jiang, Zhongxi Huang, Xuewu Chen, Rongcheng Luo, Hongbin Cai, Hairu Wang, Hui Zhang, Tao Sun, and Yunfang Zhang, an error was discovered on page 813 in the article Abstract, and on page 814 in the Introduction.

In this statement in the Abstract, "However, knockdown of FOXO1 significantly abrogated the TFP-induced apoptosis by increasing the Bcl-2 expression," the opposite was found to be true, thus "increasing" should have been changed to "decreasing." The sentence should have read: "However, knockdown of FOXO1 significantly abrogated the TFP-induced apoptosis by decreasing the Bcl-2 expression."

Similarly, on page 814, in the last paragraph of the Introduction, the statement "FOXO1 was reactivated by TFP to restore the nuclear localization and promote TFP-induced apoptosis by increasing the Bcl-2 expression" should have stated: "FOXO1 was reactivated by TFP to restore the nuclear localization and promote TFP-induced apoptosis by decreasing the Bcl-2 expression."

The online version of the article has been corrected to reflect these changes.

The authors wish to apologize for the error. 